\title{
Modulation of Oxidative Phosphorylation with IM156 Attenuates Mitochondrial Metabolic Reprogramming and Inhibits Pulmonary Fibrosis $\$$
}

\author{
Robert N. Willette, Parth Mangrolia, Stephen M. Pondell, Christopher Young Woo Lee, \\ Sanghee Yoo, Marc S. Rudoltz, Benjamin R. Cowen, and Dean J. Welsch \\ ImmunoMet Therapeutics Inc., Houston, Texas
}

Received June 24, 2021; accepted September 20, 2021

\begin{abstract}
Metabolic reprogramming of the myofibroblast plays a fundamental role in the pathogenesis of fibrosing interstitial lung diseases. Here, we characterized the in vitro and in vivo metabolic and antifibrotic effects of IM156, an oxidative phosphorylation (OXPHOS) modulator that acts by inhibiting protein complex 1 . In vitro, IM156 inhibited transforming growth factor $\beta$ (TGF $\beta$ )dependent increases in mitochondrial oxygen consumption rate and expression of myofibroblast markers in human pulmonary fibroblasts without altering cell viability or adding to TGF $\beta$ induced increases in the extracellular acidification rate. IM156 significantly increased cellular AMP-activated protein kinase (AMPK) phosphorylation and was 60 -fold more potent than metformin. In vivo, chronic oral administration of IM156 was highly distributed to major peripheral organs (i.e., lung, liver, kidney, heart) and had significant dose-related effects on the plasma metabolome consistent with OXPHOS modulation and AMPK activation. IM156 increased glycolysis, lipolysis, $\beta$-oxidation, and amino acids and decreased free fatty acids,
\end{abstract}

tricarboxylic acid cycle activity, and protein synthesis. In the murine bleomycin model of pulmonary fibrosis, daily oral administration of IM156, administered 7 days after lung injury, attenuated body/lung weight changes and reduced lung fibrosis and inflammatory cell infiltration. The plasma exposures of IM156 were comparable to well tolerated doses in human studies. In conclusion, the metabolic and antifibrotic effects of IM156 suggest that OXPHOS modulation can attenuate myofibroblast metabolic reprogramming and support testing IM156 as a therapy for idiopathic pulmonary fibrosis and other fibrotic diseases.

\section{SIGNIFICANCE STATEMENT}

Fibrosing interstitial lung diseases have a poor prognosis, and current antifibrotic treatments have significant limitations. This study demonstrates that attenuation of fibrogenic metabolic remodeling, by modulation of oxidative phosphorylation with IM156, prevents myofibroblast phenotype/collagen deposition and is a potentially effective and translational antifibrotic strategy.

\section{Introduction}

Progressive tissue fibrosis is associated with poor patient outcomes and high health care costs in a wide variety of rare and common diseases (Zhao et al., 2020), including fibrosing interstitial lung diseases (FILDs), of which idiopathic pulmonary fibrosis (IPF) is the best characterized (Kolb and Vašáková, 2019). The current US Food and Drug Administration-approved antifibrotic standard of care for these patients includes treatment with either Ofev (nintedanib) or Esbriet (pirfenidone), which act by inhibiting growth factor receptors and proinflammatory signaling, respectively, and have been shown to decrease the progression of lung dysfunction (Graney and Lee, 2018; Goldberg, 2019; Kaunisto et al., 2019). Less impressive are effects on

Funding was provided by ImmunoMet Therapeutics (Houston, TX) https://dx.doi.org/10.1124/jpet.121.000811.

S This article has supplemental material available at jpet.aspetjournals.org. survival and quality of life, as these agents have significant drug-related adverse events (i.e., gastrointestinal, liver, rash, weight loss) that require dose reductions or discontinuance in nearly $50 \%$ of patients at 1 year (Flaherty et al., 2019). New treatment options with novel mechanisms are urgently needed for patients with FILD.

Myofibroblasts, originating primarily from fibroblasts and pericytes, play an essential role in the relentless extracellular matrix remodeling and fibrogenesis that lead to loss of pulmonary function observed in patients with IPF (Horowitz and Thannickal, 2006; Meng et al., 2016; Yazdani et al., 2017; Gibb et al., 2020; Kuppe et al., 2021). The myofibroblast phenotype, distinct from the fibroblast/pericyte phenotype, includes expression and assembly of $\alpha$-smooth muscle actin-containing stress fibers and focal adhesions to allow contraction and stress activation as well as robust expression, processing, and deposition of extracellular matrix proteins, i.e., fibronectin and collagens (Zent and Guo, 2018). Critical to

ABBREVIATIONS: A.A, antibiotic-antimycotic; AMPK, AMP-activated protein kinase; BAL, bronchioalveolar lavage; COL1A1, type-1 collagen; ECAR, extracellular acidification rate; EMEM, Eagle's minimum essential medium; FILD, fibrosing interstitial lung disease; IPF, idiopathic pulmonary fibrosis; LC-MS/MS, liquid chromatography-tandem mass spectrometry; MT, Masson's trichrome; OCR, oxygen consumption rate; OXPHOS, oxidative phosphorylation; PC1, protein complex $1 ; \alpha$-SMA, $\alpha$-smooth muscle actin; TCA, tricarboxylic acid; TFI, total fluorescence intensity; TGF $\beta$, transforming growth factor $\beta$. 
the development and maintenance of the myofibroblast phenotype is a complex process of metabolic reprogramming that enables amphibolic pathways to meet the energetic and synthetic requirements of an active myofibroblast (Hua et al., 2020). Metabolic remodeling in the myofibroblast includes enhanced aerobic glycolysis, anaplerosis, and oxidative phosphorylation (OXPHOS); interruption of these metabolic pathways may have antifibrotic effects (Win et al., 2012; Chen et al., 2014; Bernard et al., 2015; Bernard et al., 2018; Bueno et al., 2020).

The acquisition and maintenance of the myofibroblast phenotype is driven by extracellular mediators liberated after tissue injury, inflammation, and/or stretch - of which the most prominent and well characterized is transforming growth factor $\beta(\mathrm{TGF} \beta)$. Often referred to as the "master regulator," TGF $\beta$ exerts context-dependent morphogenic, inflammogenic, and fibrogenic actions through activin-like kinase receptormediated canonical and noncanonical signaling pathways (Meng et al., 2016; Stewart et al., 2018). The upregulation of $\mathrm{TGF} \beta$ has been described in numerous fibrotic disorders, and its levels have been correlated with the fibrotic burden and severity of disease. TGF $\beta$ initiates metabolic remodeling as a necessary step in fibroblast to myofibroblast transition by upregulating metabolic transcription factors, enzymes, transporters, and mitochondrial mass (Bernard et al., 2015; Bueno et al., 2020; Gibb et al., 2020; Hua et al., 2020). Direct inhibition of TGF $\beta$ signaling has profound antifibrotic effects in preclinical models, but it is also associated with serious toxicities that preclude its direct targeting as a therapeutic strategy.

In the present study we have focused on the role of OXPHOS in the myofibroblast phenotype, metabolism, and fibrosis by evaluating the in vitro and in vivo effects of IM156, a selective mitochondrial protein complex 1 (PC1) modulator (Izreig et al., 2020). IM156 is a newly developed biguanide molecule that has been optimized for its potency, bioavailability, cellular distribution, and safety. IM156 is significantly more potent than metformin in inhibiting PC1 catalytic activity and associated mitochondrial respiration (Izreig et al., 2020). These properties of IM156 allow a more thorough evaluation of the role of OXPHOS in fibrosis than previous compounds that were limited by potency and/or toxicities. Our findings illustrate the critical role played by OXPHOS in the myofibroblast phenotype induced by TGF $\beta$ and in fibrosis and demonstrate the therapeutic potential of IM156 for the treatment of IPF and other fibrotic diseases.

\section{Materials and Methods}

\section{Fibroblast Culture Conditions}

WI-38, human embryonic lung fibroblasts, were obtained from American Type Culture Collection (CCL-75; Manassas, VA) and grown in Eagle's minimum essential medium (EMEM) supplanted with $10 \%$ FBS and $1 \%$ antibiotic-antimycotic (A.A; 100 units $/ \mathrm{ml}$ of penicillin, $100 \mu \mathrm{g} / \mathrm{ml}$ of streptomycin, and $0.25 \mu \mathrm{g} / \mathrm{ml}$ of amphotericin B) at $37^{\circ} \mathrm{C}$ in $5 \% \mathrm{CO}_{2}$. Cells were passaged a maximum of 10 times before use in assays. Once WI-38 fibroblast cultures reached 80-90\% confluency, cells were harvested for subsequent experiments.

\section{Cellular Metabolism}

Ten thousand fibroblasts per well were seeded into a Seahorse XF96 microplate (Agilent; 102416-100) under growth conditions for 24 hours. Cells were pretreated with or without varying concentrations of IM156 for 2 hours in EMEM supplanted with 0.5\% FBS and 1\% A.A at $37^{\circ} \mathrm{C}, 5 \% \mathrm{CO}_{2}$. Cells were then stimulated with differentiation medium [5 ng/ml TGF $\beta 1$ (R\&D Systems), $100 \mu \mathrm{M}$ ascorbic acid (Sigma), $37.5 \mathrm{mg} / \mathrm{ml}$ Ficoll 70 (Sigma), $25 \mathrm{mg} / \mathrm{ml}$ Ficoll 400 (Sigma), $0.5 \%$ FBS, $1 \%$ A.A in EMEM] for 24 hours at $37^{\circ} \mathrm{C}, 5 \% \mathrm{CO}_{2}$. Nonstimulated control cells were treated with EMEM and supplanted with $0.5 \%$ FBS and 1\% A.A, with or without IM156. The oxygen consumption rate $(\mathrm{OCR})$ and extracellular acidification rate (ECAR) were measured simultaneously in fibroblasts, and during the time course of the assay, metabolic modulators were used to perform a mitochondrial stress test at specific time points according to the manufacturer's instructions (Seahorse XF Cell Mito Stress Test Kit; Agilent; 103015100) as follows: 1) ATP synthase (complex V) inhibition with oligomycin allowed assessment of OCR linked to cellular ATP production. 2) The uncoupling ionophore carbonyl cyanide-4-(trifluoromethoxy)phenylhydrazone was used to determine maximal OCR. 3) Rotenone and antimycin (complex I and complex III inhibitors) were used to abolish mitochondrial OCR. The OCR or ECAR signal per well was normalized to total viable cells per well. Edge wells were excluded from data analysis. Oxygen consumption attributed to ATP-linked production, proton leak, maximum and reserve capacity, and nonmitochondrial respiration was calculated as described previously (Hill et al., 2012). Cell viability per well was measured via CyQuant (Invitrogen; C7026) using a Cytation 5 multimode reader (BioTek).

\section{Myofibroblast Cellular Assay}

WI-38 cells were prepared as above and seeded at a density of 15,000 cells per well in a black-walled, clear bottom 96-well culture plate (29444-008; VWR, Radnor, PA) under growth conditions for 24 hours to reach confluency. Cells were pretreated with or without IM156 (varying concentration) for 2 hours (when measuring fibroblast to myofibroblast transition) or 24 hours (when measuring collagen deposition) in EMEM containing $0.5 \% \mathrm{FBS}$ and $1 \%$ A.A at $37^{\circ} \mathrm{C}, 5 \% \mathrm{CO}_{2}$. Cells were then stimulated with TGF $\beta$-containing differentiation medium $[5 \mathrm{ng} / \mathrm{ml}$ TGF $\beta 1$ (R\&D Systems), $100 \mu \mathrm{M}$ ascorbic acid (Sigma), $37.5 \mathrm{mg} / \mathrm{ml}$ Ficoll 70 (Sigma), 25 mg/ml Ficoll 400 (Sigma), 0.5\% FBS, 1\% A.A in EMEM] for 24 hours (when measuring collagen) or 48 hours (when measuring fibroblast to myofibroblast transition) at $37^{\circ} \mathrm{C}, 5 \% \mathrm{CO}_{2}$. This culturing technique is commonly referred to as "scar in a jar" as it allows robust collagen deposition (Chen et al., 2009).

\section{Immunocytochemistry for Collagen, $\alpha$-Smooth Muscle Actin, and Cell Count Measurement}

Fibroblasts were washed with PBS and then fixed in ice-cold methanol for 5 minutes followed by another PBS wash. After blocking with $3 \%$ bovine serum albumin (Sigma) for 60 minutes at room temperature, fibroblasts were incubated with their respective primary antibodies against type- 1 collagen (Sigma; C2456) or $\alpha$-smooth muscle actin $\left(\alpha-S M A ;\right.$ Sigma; A2547) at 1:1000 in PBS overnight at $4^{\circ} \mathrm{C}$. After washing with PBS, fibroblasts were incubated with their respective secondary antibodies (AlexaFluor488 or AlexaFluor594; ThermoFisher, A-11001 or A11032) at 1:500 in PBS for 1 hour at room temperature protected from light. Total fluorescence intensity (TFI), for collagen or $\alpha$-SMA, and cell count per well were quantified from images using Image Statistics and Cellular Analysis algorithms, respectively, in Gen5 (BioTek; version 3.05) software. Background TFI (collagen or $\alpha$-SMA fluorescence from fibroblasts treated in absence of TGF $\beta$ and IM156) was subtracted from TFI per well. TFI per well was normalized to TFI of fibroblasts treated with TFG- $\beta$ in absence of IM156 and corrected by cell count. Edge wells were excluded from data analysis.

\section{Study Approval}

All animal protocols were approved by the St. Louis University's Institutional Animal Care and Use and were conducted in an Association for the Assessment and Accreditation of Laboratory Animal Care-accredited animal facility in the Department of Comparative Medicine. All study procedures were performed in accord with Guide for the Care and Use of 
Laboratory Animals as adopted and promulgated by the US National Institutes of Health. The doses of IM156 used in these studies did not exhibit toxicologic findings in 28-day rodent and dog safety studies.

\section{IM156 Quantification and Isolation Techniques}

Plasma and tissue concentrations of IM156 were determined in $\mathrm{BALB} / \mathrm{c}$ female mice (body weight $>20 \mathrm{~g}$, SIPPR/BK, Laboratory Animal Ltd., Shanghai) and in male Wistar rats (body weight $250 \mathrm{~g}$, Janvier Laboratories, France). A standard liquid chromatography-tandem mass spectrometry (LC-MS/MS) method was developed for quantification of IM156 using IM156 calibration standards and spiked quality control samples. Plasma samples $(0.05 \mathrm{ml})$ were transferred to tubes; then a $250 \mu$ internal standard solution (200 ng/ml IM156 in methanol) was added to it. After vortexing for 1 minute and centrifuging for 5 minutes at $15000 \mathrm{rpm}, 100 \mu \mathrm{l}$ aliquots of supernatant were transferred to 96 -well plate for LC-MS/MS injection $(1 \mu \mathrm{l})$. The lower limit of quantification for plasma was $1 \mathrm{ng} / \mathrm{ml}$. Tissues samples were homogenized by adding saline ( $1 \mathrm{~g}$ tissue: $5 \mathrm{ml}$ saline), and the homogenate $(50 \mu \mathrm{l})$ was transferred to tubes with $250 \mu \mathrm{l}$ of the IM156 internal standard working solution (as above). After vortexing for 1 minute and centrifuging for 5 minutes at $15,000 \mathrm{rpm}, 100 \mu \mathrm{l}$ aliquots of supernatant were transferred to 96 -well plate for injection $(1 \mu \mathrm{l})$. The lower limit of quantification for tissue was $5 \mathrm{ng} / \mathrm{ml}$.

Mitochondrial Isolation. Mitochondrial and cytosolic cellular fractions were prepared using the Qproteome Mitochondria Isolation Kit (Qiagen, Hilden, Germany) according to the manufacturer's instructions. All steps were performed at $4{ }^{\circ} \mathrm{C}$ using precooled buffers, and protein fractions were snap-frozen in liquid nitrogen, stored at $-80^{\circ} \mathrm{C}$, and prepared for LC-MS/MS as above.

\section{Plasma Metabolomics}

Untargeted metabolomic profiling was performed at Metabolon Inc. (Morrisville, NC) using a combination of liquid chromatography-mass spectrometry methods as described by Evans et al. (2014). All methods used a Waters ACQUITY UPLC and a Thermo Scientific Q-Exactive high resolution/accurate mass spectrometer interfaced with a heated electrospray ionization (HESI-II) source and Orbitrap mass analyzer operated at 35,000 mass resolution. Briefly, the sample extract was dried then reconstituted in solvents compatible with each of the four methods. Each reconstitution solvent contained a series of standards at fixed concentrations to ensure injection and chromatographic consistency. Based on Metabolon Inc. protocols and previously published methods, the first aliquot was analyzed using acidic positive ion conditions, chromatographically optimized for more hydrophilic compounds. The second aliquot was also analyzed using acidic positive ion conditions; however, it was chromatographically optimized for more hydrophobic compounds. The third aliquot was analyzed using basic negative ion optimized conditions and a separate dedicated C18 column. The fourth aliquot was analyzed via negative ionization after elution from a HILIC column (Waters UPLC BEH Amide $2.1 \times 150 \mathrm{~mm}, 1.7 \mu \mathrm{m}$ ) using a gradient consisting of water and acetonitrile with $10 \mathrm{mM}$ Ammonium Formate, $\mathrm{pH}$ 10.8. The mass spectrometry analysis alternated between mass spectrometry and data-dependent muti-stage mass spectrometry $\left(\mathrm{MS}^{\mathrm{n}}\right)$ scans using dynamic exclusion. The scan range varied slightly between methods but covered $70-1000 \mathrm{~m} / \mathrm{z}$. Raw data files were archived and extracted as described below. Metabolites were identified by comparison with a referenced library of chemical standards, and area-under-the-curve analysis was performed for peak quantification and normalized to day median value. To ensure high quality of the data set, control and curation processes were subsequently used to ensure true chemical assignment and remove artifacts and background noise. Metabolites were scaled by run-day medians and log-transformed before statistical analysis.

\section{Bleomycin-Induced Pulmonary Fibrosis}

Sixty male C57BL/6 mice, 6-7 weeks of age, were purchased from Taconic Laboratories (Rensselaer, NY) and allowed to acclimate for 1 week prior to experimentation. Only males were used because bleomycin-induced lung fibrosis exhibits significant sexual dimorphism in mice (Redente et al., 2011). Body weights were recorded one day prior to study initiation and on day 6 after bleomycin administration. To induce pulmonary fibrosis, mice in groups $2-5$ were administered $70 \mu \mathrm{l}$ of $1.5 \mathrm{U} / \mathrm{kg}$ bleomycin in PBS (C-61703-323-22, lot number D011495AA, Hospira) via oropharyngeal administration on day 0 . Animals from group 1 were administered $70 \mu \mathrm{l}$ of saline by the oropharyngeal route, instead of bleomycin, and served as sham control (Walters and Kleeberger, 2008). On day 6, 40 bleomycin-treated animals were randomized into groups based on percent change in initial body weight such that mean percent body weights were similar for the different groups (Supplemental Table 1). Remaining animals with lower or higher body weight were not included into the study. Food and water were provided ad libitum, with a light/ dark cycle of 12 hours.

Study Design. On day 0, all mice in groups $2-5$ received a 1.5 $\mathrm{U} / \mathrm{kg}$ oropharyngeal administration of bleomycin to induce pulmonary fibrosis. Animals in group 1 were not administered with bleomycin but instead received a single dose of saline via the oropharyngeal route, and they were considered sham control mice (Supplemental Table 1). Therapeutic treatment was initiated on day 7 after disease induction and continued until study end. Study animals were harvested on day 21 after bleomycin administration. Endpoint readouts were measured from mice in the experimental groups on day 21 .

Clinical Observations and Body Weights. Individual animals were monitored daily for clinical observations, including general activity levels and morbidity. Body weights were recorded three times a week during the study period.

Compounds and Treatment Regimen. The amount of drug administered to animals was calculated based on average body weight of the groups on day 6 and day 13. IM156 was diluted in water, and 30 and $10 \mathrm{mg} / \mathrm{kg}$ doses of IM156 were formulated once a week. Animals were administered $250 \mu \mathrm{l}$ once daily via oral gavage. Pirfenidone was formulated weekly in $0.5 \%$ methylcellulose as a vehicle. Vehicle (0.5\% methylcellulose) was prepared fresh weekly. Animals from group 5 were treated with $100 \mathrm{mg} / \mathrm{kg}$ of pirfenidone twice daily via oral route in volume of $100 \mu \mathrm{l}$ per dose. The dose of pirfenidone was based on previous literature studies (Oku et al., 2008). The dosing regimen for test compounds was initiated on day 7 after bleomycin administration and continued until the day of harvest on day 21. Animals were harvested within 2 to 4 hours after final dose.

Harvest Procedures and Blood Collection. Upon study termination on day 21, study animals were anesthetized with isoflurane inhalant anesthesia, and terminal cardiac blood collection from each animal was performed using a $1 \mathrm{ml}$ syringe with a 23 -gauge $3 / 4$ " needle. Promptly after collection, blood was transferred to EDTA tubes. Death was ensured by cervical dislocation. Plasma was separated by centrifugation, transferred to a separate tube, and stored at $-80^{\circ} \mathrm{C}$. The internal organs of each animal were exposed and observed for abnormalities. Each lung was dissected from the animal and weighed. Postcaval lobe was separated and snap frozen. The bronchioalveolar lavage (BAL) fluid was collected by lavaging the lung twice with 0.5 ml Hanks' balanced salt solution (VWR). After collection of BAL fluid, whole lungs were inflated with $10 \%$ neutral buffered formalin, fixed in $10 \%$ neutral buffered formalin, and transferred for histopathology.

BAL Cell Count. After collection, the BAL fluid from each mouse was centrifuged at $1,000 \mathrm{rpm}$ at $4^{\circ} \mathrm{C}$ for 5 minutes. The BAL fluid supernatant was transferred into three separate tubes with aliquots of $200 \mu \mathrm{l}$ aliquots each, snap frozen on dry ice, and stored at $-80^{\circ} \mathrm{C}$. The BAL cell pellets were then resuspended in $2 \mathrm{ml}$ of $1 \times$ Pharm Lyse buffer (BD Biosciences, San Jose, CA) to lyse red blood cells. PBS supplemented with $2 \%$ FBS was added to stop further cell lysis. The BAL cells were again centrifuged and finally suspended in $250 \mu \mathrm{l}$ of PBS. Viable cells were counted using a hemocytometer and trypan blue staining and recorded for each mouse. The BAL cells were spun down on the slides using Cytospin. Slides were fixed and stained with May-Gruland-Geimsa stain and differential counts were recorded from each slide. 


\section{Histopathology}

Whole lungs from each mouse were paraffin-embedded in a single block. Two slides from each block were sectioned to the depth of the mainstem bronchi (near the center of each lobe) and stained with either H\&E or Masson's trichrome (MT). Glass slides were evaluated using light microscopy by a board-certified veterinary pathologist (HistoTox Laboratories, Inc., Boulder, CO). Lung sections were scored according to the modified Ashcroft scale (Hübner et al., 2008). Briefly, scores for five representative 200× microscopic fields per sample were averaged to obtain a mean score for each animal (Supplemental Table 2).

Infiltration/aggregation of mononuclear cells (macrophages and lymphocytes) and neutrophilic infiltrates were scored in H\&E-stained sections and increased collagen (fibrosis) was scored in MT-stained sections. These features were graded for severity $0-5(0=$ not present/ normal, $1=$ minimal, $2=$ mild, $3=$ moderate, $4=$ marked, $5=$ severe) (Crissman et al., 2004).

\section{Statistical Analysis}

Graphs, concentration response curves, and statistical analysis were constructed using GraphPad software (version 8.4.2, La Jolla, CA). Statistical comparisons were performed using one-way ANOVA with Bonferroni's multiple-comparison test or unpaired $t$ tests where appropriate. Two-way ANOVA with Dunnett's test for multiple comparisons was used for comparing time-course parameters. A principal component analysis was used to examine log-transformed data.

\section{Results}

\section{Effects of IM156 on Metabolic Reprogramming Associated with Fibroblast to Myofibroblast Transition}

The incubation of human pulmonary fibroblasts with TGF $\beta$ ( $5 \mathrm{ng} / \mathrm{ml}$ ) for 24 hours significantly increases the basal OCR and ECAR (Fig. 1, A and C) as well as ATP-linked respiration
A

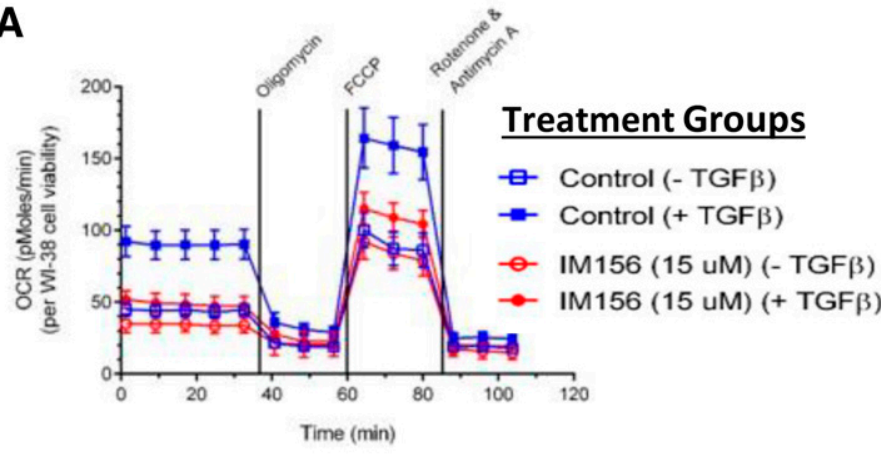

C

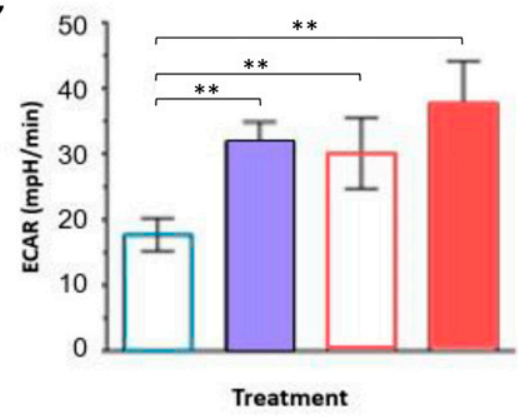

E

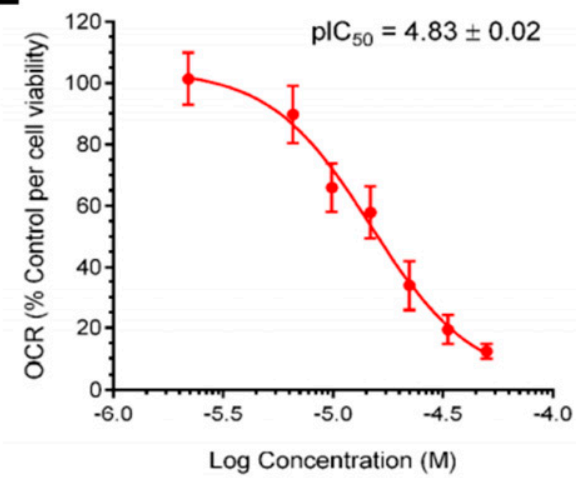

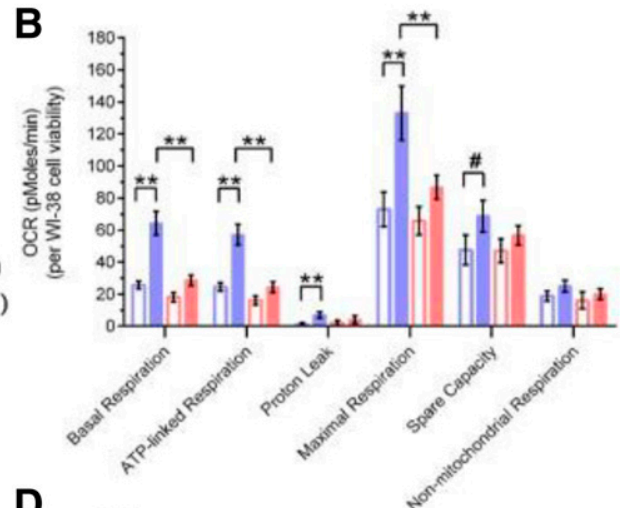

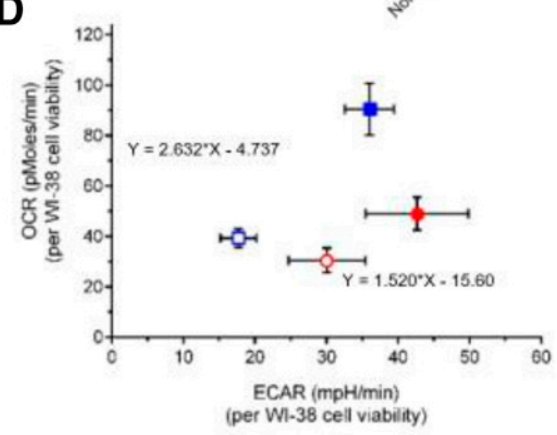

$\mathbf{F}$

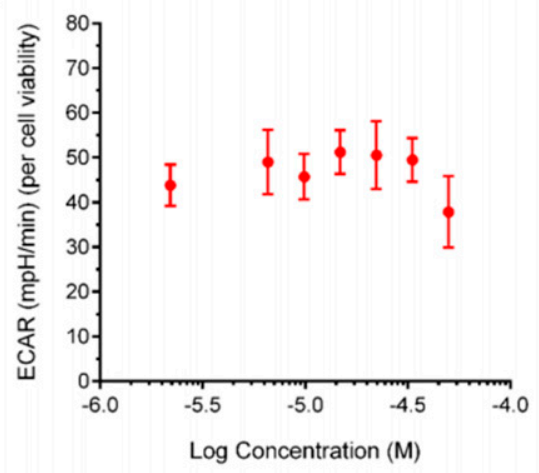

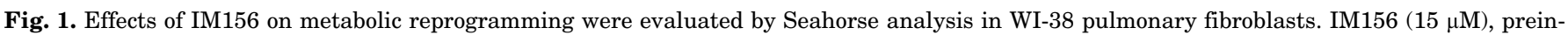

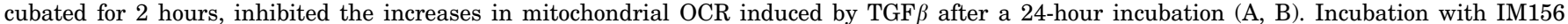

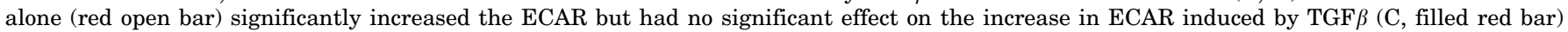

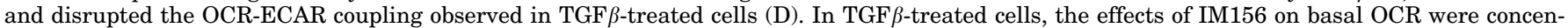

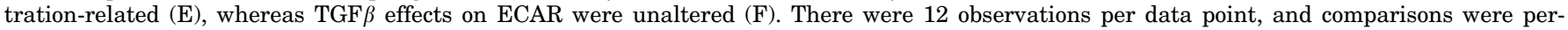

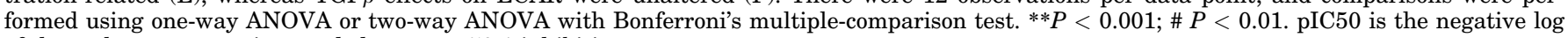
of the molar concentration needed to cause $50 \%$ inhibition. 
$(P<0.001)$, proton leak $(P<0.001)$, maximal mitochondrial respiration $(P<0.001)$, and spare mitochondrial capacity $(P<$ 0.002) observed during mitochondrial stress testing (Fig. 1B). The increases in OCR and ECAR were tightly coupled (Fig. 1D) and consistent with the effects of TGF $\beta$ to enhanced both aerobic glycolysis and OXPHOS (an energetic phenotype).

Preincubation of pulmonary fibroblasts with IM156 $(15 \mu \mathrm{M})$ alone modestly reduced basal and ATP-linked OCR and increased ECAR (Fig. 1, A-C). In contrast, IM156 abolished the effects of TGF $\beta$ on OCR (Fig. 1, A and B), and the effects of IM156 and TGF on ECAR were not additive (Fig. 1, C, D, and F). The effects of IM156 on TGF-induced increases in OCR were concentrationdependent with an $\mathrm{IC}_{50}$ of $14.7 \pm 0.1 \mu \mathrm{M}$ (Fig. $1 \mathrm{E}$ ) and are consistent with attenuation of TGF $\beta$ activation of OXPHOS without obvious differences in glycolysis.

Effects of IM156 on the Myofibroblast Phenotype Induced by TGF $\boldsymbol{\beta}$. To determine the effect of IM156 on the fibroblast to myofibroblast transition, the expression of $\alpha$-SMA and extracellular collagen deposition was evaluated in human pulmonary fibroblast cell culture grown to confluency in a crowding medium (Chen et al., 2009). Incubation of fibroblasts with TGF $\beta(5 \mathrm{ng} / \mathrm{ml})$ induced robust collagen deposition at 24 hours and $\alpha$-SMA expression at 48 hours (Fig. 2A). Preincubation with IM156 caused a concentration-dependent inhibition of both $\alpha$-SMA expression and type-1 collagen (COL1A1) deposition
(Fig. 2, A-C) but had little or no significant effect on cell count (Fig. 2D). The similar potencies of IM156 in the OCR, $\alpha$-SMA, and COL1A1 assays $\left(\mathrm{IC}_{50}\right.$ values $\left.13.2-21.3 \mu \mathrm{M}\right)$ suggest a common underlying mechanism of action.

IM156 Tissue Distribution, Subcellular Localization, and Target Engagement. A repeat-dose study was conducted to evaluate tissue and plasma levels of IM156 in $\mathrm{BALB} / \mathrm{c}$ mice. Animals received IM156 (15 mg/kg. p.o., every other day) with plasma and tissue collected for analysis 4 hours after IM156 administration on days 1, 7, and 15 . The concentrations of IM156 in plasma and brain were similar; however, tissue levels of IM156 were 30-80-fold greater in lung, liver, and kidney versus plasma (Fig. 3, A and B). Tissue concentrations were reproducible and consistent over time. Similar dose-related plasma and tissue results were also observed in rats after both intraperitoneal and oral repeated daily doses (Supplemental Fig. 1). The effects of dose on the tissue distribution and concentration of IM156 were examined in a separate arm of the study, where mice received either 15 or $60 \mathrm{mg} / \mathrm{kg}$ (p.o., every other day) for 29 days (Fig. 3, C and D). The tissue distribution of IM156 was dose-related in all tissues examined (Fig. 3C). With the possible exception of the liver the tissue to plasma ratio of IM156 concentration was similar at both high and low doses (Fig. 3D).

The subcellular distribution of IM156 was evaluated in subcellular fractions prepared from MCF7 breast cancer cells. After
A

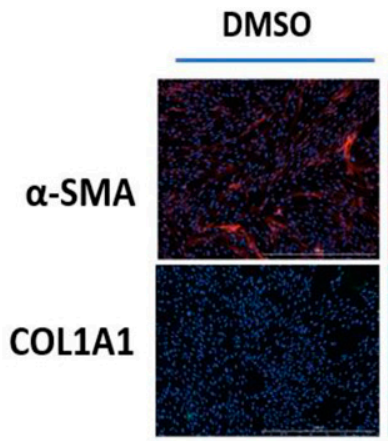

$(-)$ TGFB, (-)IM156)

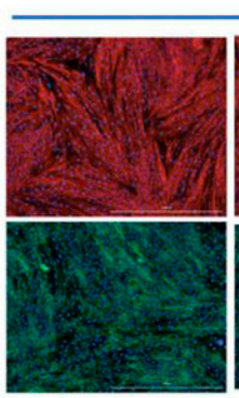

0
C

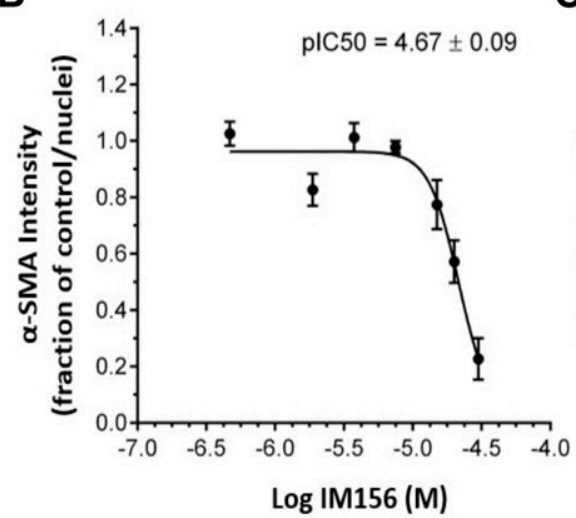

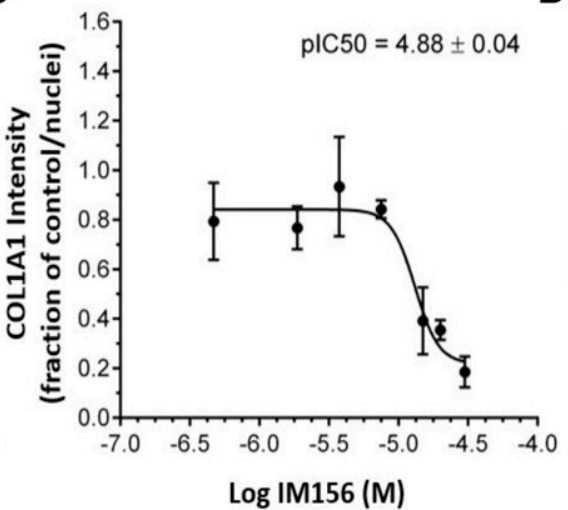

$\operatorname{TGF} \beta(5 \mathrm{ng} / \mathrm{ml})+\operatorname{IM} 156(\mu \mathrm{M})$

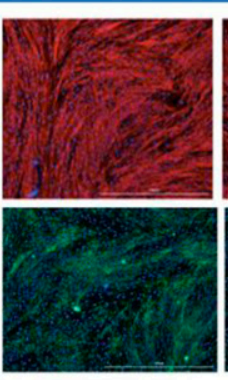

7.5

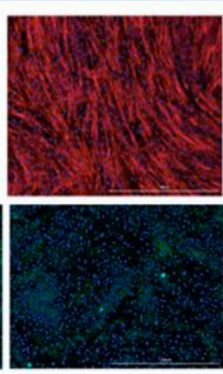

15

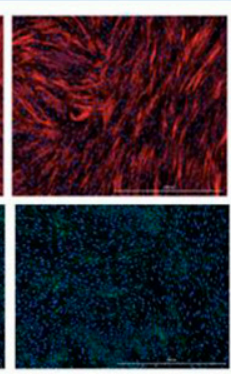

20

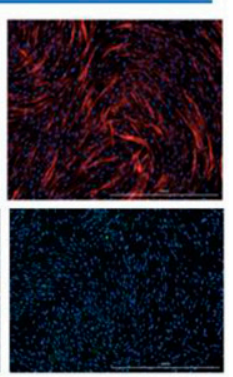

30

Fig. 2. The effects of IM156 on the myofibroblast phenotype were evaluated in WI-38 pulmonary fibroblasts stimulated with TGF $\beta$ ( 5 ng/ml). (A) Incubation with TGF $\beta$-induced expression of $\alpha$-SMA was measured at 48 hours, and COL1A1 deposition was measured at 24 hours. Co-incubation with IM156 inhibited both $\alpha$-SMA expression and COL1A1 deposition in a dose-dependent manner. (B, C) IM156 had equivalent potencies in both the $\alpha$-SMA (B) and collagen (C) assay. (D) The fibroblast cell count, based on nuclei, was preserved at all concentrations of IM156. The data are means \pm S.D. $(n=3-6)$. Statistical comparisons were performed using one-way ANOVA with Bonferroni's multiple-comparison test. 
A

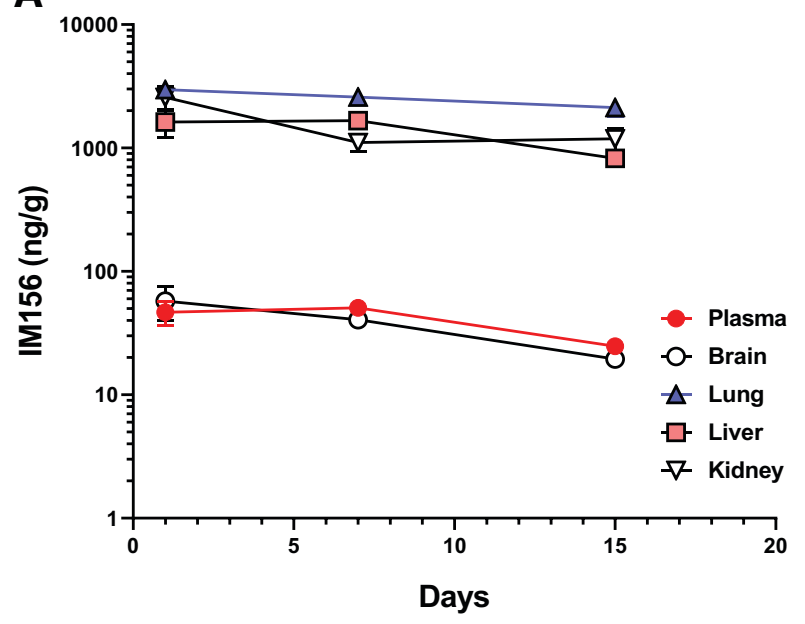

C

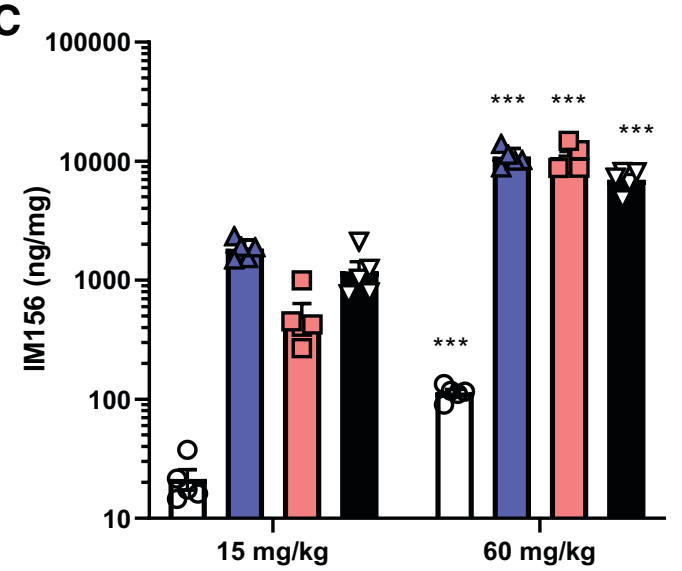

B

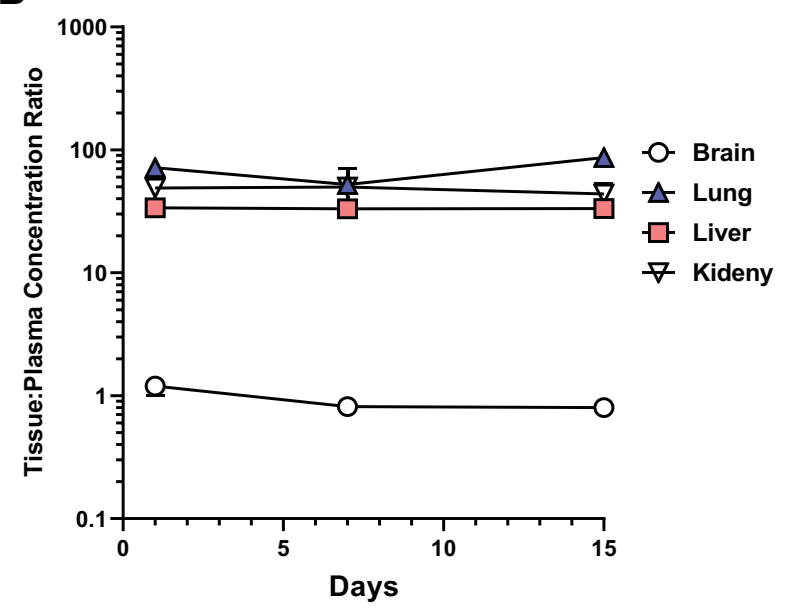

a 1-hour incubation with IM156, IM156 preferentially distributed ( $>3$-fold) to the mitochondria versus cytosol (Fig. 4). Similar data were generated after incubation for 18 hours. These results confirm previous mitochondrial distribution studies of select biguanides (Dykens et al., 2008; Bridges et al., 2014).

These data suggest that a $15 \mathrm{mg} / \mathrm{kg}$ oral dose of IM156 should result in IM156 concentrations of 5-15 $\mu \mathrm{M}$ in the lung, liver, and kidney that are likely to be higher at the mitochondrial site of action in these tissues. Based on these estimates, IM156 target engagement studies were performed using chronic daily oral doses of 10 and $30 \mathrm{mg} / \mathrm{kg}$.

Systemic Metabolic Effects of IM156. Given the selective effects of IM156 to modulate OXPHOS, a plasma metabolomic study was conducted to assess in vivo target engagement. Plasma metabolomic analysis was performed on time-controlled plasma samples obtained from CD-1 mice after 10 days of once daily oral gavage with IM156 (10 and $30 \mathrm{mg} / \mathrm{kg}$ ) or vehicle (water $250 \mu \mathrm{l}$ ). A total of 878 metabolites met the manufacturer's quality assurance requirements (793 identified and 85 unnamed). A significant change was observed in $44 \%$ of the plasma metabolites in mice treated with IM156 (30 mg/kg) compared with the vehicle group on day 10 . These effects were dose-related; only $9 \%$ of the plasma metabolome was significantly altered in the IM156 low-dose group (10 mg/kg). Consistent with these results, a principle component analysis suggested two distinct clusters composed of IM156 (30 $\mathrm{mg} / \mathrm{kg}$ ) and vehicle treatment and an intermediate cluster defined by the $10 \mathrm{mg} / \mathrm{kg}$ dose of IM156 (Fig. 5). Analyses of the plasma metabolome at the pathway level suggested significant dose-related effects of IM156 on cytosolic and mitochondrial metabolism in the liver and other highly metabolic tissues (Fig. 6). Some of the general metabolic themes after treatment of mice with IM156 included the following:

1) Reduced glucose and elevated phosphate, pyruvate, lactate, tricarboxylic acid (TCA) cycle intermediates, anaplerotic 

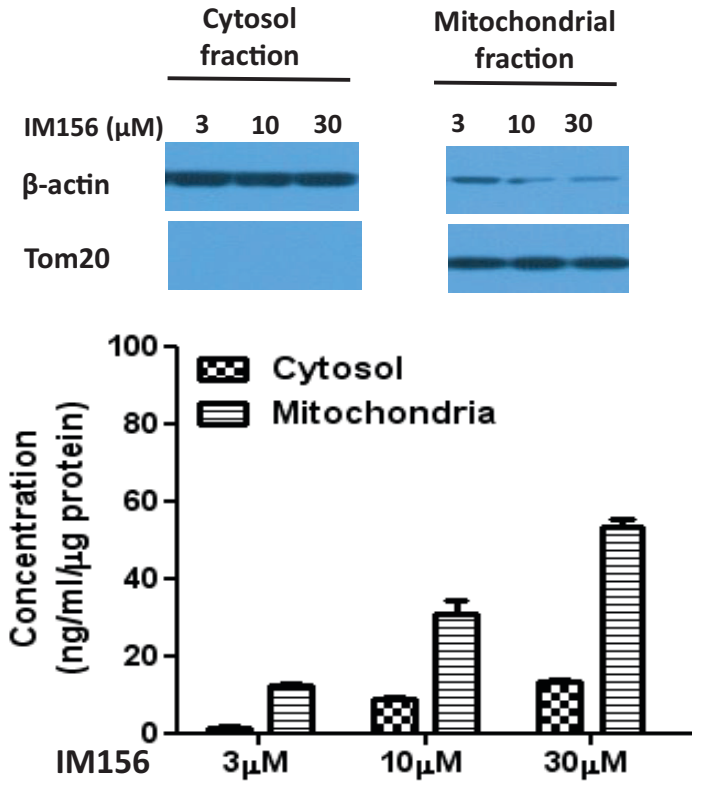

Fig. 4. Cytosolic and mitochondrial concentrations of IM156 were determined in subcellular fractions of MCF7 cells after 1-hour incubation with IM156. Cytosolic and mitochondrial markers [ $\beta$-actin and the mitochondrial outer membrane translocase (Tom20), respectively] were measured by Western blot analysis. All values were expressed as mean \pm S.E.M.

amino acids, and branched chain amino acids. These findings are consistent with increased glucose uptake and glycolysis and decreased TCA cycle and OXPHOS activity.

2) Decreased free fatty acids and increased ketones indicative of increased $\beta$-oxidation and a surplus of acetyl-CoA.

3) Elevated levels of amino acids in the context of increased dimethylarginine, acetylated lysine, and hydroxy proline metabolites, suggesting changes in protein homeostasis indicative of enhanced protein/collagen breakdown.

4) Evidence of reduced eicosanoids and glutathione recycling/ demand suggesting decreased reactive oxygen species and inflammation.

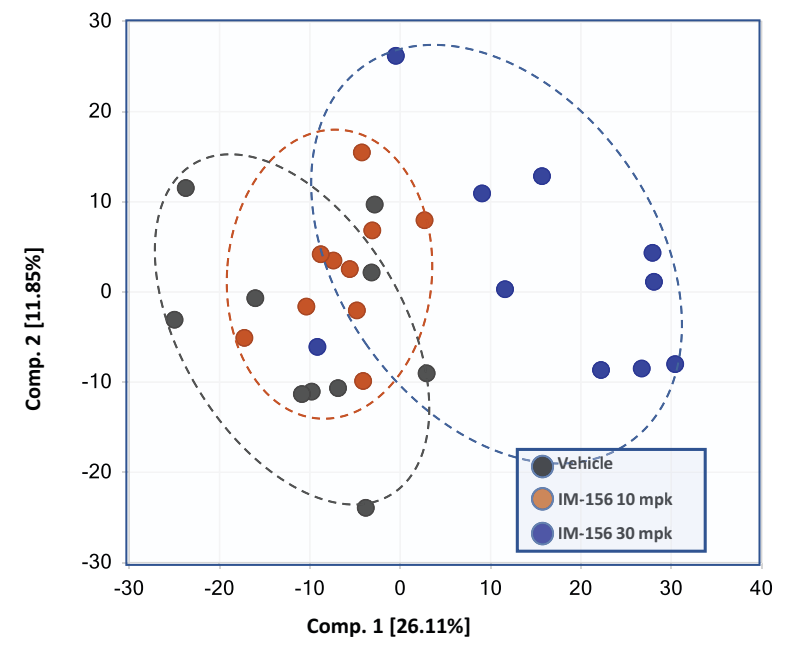

Fig. 5. Principal component scatterplots based on 878 plasma metabolites identified in the 10-day treatment groups (vehicle, IM156 $10 \mathrm{mg} / \mathrm{kg}$, and IM156 $30 \mathrm{mg} / \mathrm{kg}$ ) and putative clusters. Clusters at the extremes are define by the vehicle and IM156 (30 mg/kg) groups, and an intermediate cluster is defined by the low-dose IM156 group $(10 \mathrm{mg} / \mathrm{kg}) . \mathrm{mpk}$ $(\mathrm{mg} / \mathrm{kg})$.
These findings are summarized in a working model (Fig. 7) and demonstrate the metabolic effects of IM156 and previously described adaptation associated with activation of AMPactivated protein kinase (AMPK) (Chen et al., 2014; Lee et al., 2016; Brockhoff et al., 2017; Garcia and Shaw, 2017; Li and Chen, 2019). It is noteworthy that IM156 effectively increases phosphorylation of AMPK in cellular assays and is approximately 60-fold more potent than metformin (Supplemental Fig. 2).

Effects of IM156 in the Murine Bleomycin-Induced Model of Pulmonary Fibrosis. Based on cellular potency determinations, tissue/cellular distribution, and target engagement studies, two doses (10 and $30 \mathrm{mg} / \mathrm{kg}$, p.o., every day) were selected for evaluation of the antifibrotic effects of IM156 in a murine bleomycin-induced model of pulmonary fibrosis. These doses were without adverse effect in 28-day rodent and canine safety studies (data not shown).

All animals administered bleomycin to induce pulmonary fibrosis exhibited abrupt weight loss over the first 7 days (Fig. 8A). Body weight vacillated somewhat but continued to decline throughout the study in animals receiving bleomycin alone. Treatment with IM156 beginning on day 7 attenuated the body weight loss, and a significant improvement in body weight was observed in the IM156 high-dose group on study days 19 and $21(P=0.05$ and $P=0.03$, respectively; Fig. 8A). Bleomycin administration also increased lung weights (absolute or normalized) at day 21 , and delayed treatment with IM156 or pirfenidone (comparator) reduced lung weight as follows: IM156 $(30 \mathrm{mg} / \mathrm{kg}$, daily) > pirfenidone $(100 \mathrm{mg} / \mathrm{kg}$, twice daily) > IM156 (10 mg/kg, daily) (Fig. 8B). Treatments were well tolerated, and no mortality was observed. These data confirm and extend earlier studies in this model where IM156 was administered every other day in a prophylactic dosing paradigm.

Total viable leukocyte counts were significantly increased in BAL fluid of the bleomycin-treated vehicle control group when compared with the sham control no-bleomycin group $(130,000 \pm 19,500$ per $\mathrm{ml}$ versus $29300 \pm 17000, P<0.0001)$. The average leukocyte counts in BAL fluid were variably reduced by treatments and not statistically significant.

The magnitude of fibrosis was quantified using modified Ashcroft $(0-8)$ and MT $(0-5)$ scoring, and cellular infiltration was scored (0-5), as described in Materials and Methods. Fibrosis and cellular infiltration were absent in control animals not exposed to bleomycin and were most severe in the bleomycin-treated group that received vehicle (Fig. 8, C-E). IM156 treatment significantly reduced the average Ashcroft, MT, and cellular infiltration scores in a dose-related manner (Fig. 8, C-E). Reductions in histopathology scores observed in the pirfenidone treatment group were similar to those observed in the low-dose IM156 group.

Whole lungs from each mouse were paraffin-embedded, and H\&E- and MT-stained slides were prepared from sections obtained at the mainstem bronchi near the center of each lobe (Fig. 9). Bleomycin-induced pulmonary fibrosis was characterized by expansion of alveolar walls, formation of fibrous bands or nodular masses, and partial to complete obliteration of lung architecture by fibrous connective tissue (collagen). In areas of fibrosis, alveoli were sometimes lined by hypertrophied and hyperplastic type II pneumocytes and variably contained foamy alveolar macrophages or pale eosinophilic edema fluid. Infiltrates and aggregates of mononuclear cells (primarily lymphocytes and macrophages) were present in areas of fibrosis. Clusters of neutrophils were sometimes observed in perihilar regions or within the interstitium and alveoli, usually 


\begin{tabular}{|c|c|c|c|}
\hline \multirow{2}{*}{$\begin{array}{c}\text { Pathways } \\
\text { (sub-pathways) }\end{array}$} & \multirow{2}{*}{ Biochemical Name } & \multicolumn{2}{|c|}{ Day- 10} \\
\hline & & $\begin{array}{c}\text { IM156 } 10 \mathrm{mpk} \\
\text { Yehicle } \\
\end{array}$ & \begin{tabular}{|c|} 
IM156 30 mpk \\
Yehicle \\
\end{tabular} \\
\hline \multirow{4}{*}{$\begin{array}{c}\text { Glycolysis } \\
\text { [Gluconeogenesis } \\
\text { and Pyruvate } \\
\text { Metabolism] }\end{array}$} & glucose & 0.96 & 0.76 \\
\hline & \begin{tabular}{|l|} 
pyruvate \\
\end{tabular} & 1.11 & 1.88 \\
\hline & Iactate & 1.1 & 1.72 \\
\hline & & & \\
\hline \multirow{5}{*}{ TCA Cycle } & itaconate & 2.26 & 3.62 \\
\hline & fumerate & 1.29 & 2.58 \\
\hline & \begin{tabular}{|l|} 
malate \\
\end{tabular} & 1.25 & 2.37 \\
\hline & citraconatelglutaconat & 0.94 & 1.58 \\
\hline & \begin{tabular}{|l|} 
alpha-ketoglutarate \\
\end{tabular} & 0.99 & 1.5 \\
\hline \multirow{2}{*}{$\begin{array}{c}\text { Oxidative } \\
\text { Phosphorylation }\end{array}$} & phosphate & 0.94 & 1.25 \\
\hline & & & \\
\hline \multirow{5}{*}{$\begin{array}{l}\text { Fatty Acid } \\
\text { Oxidation }\end{array}$} & carnitine & 0.88 & 0.83 \\
\hline & \begin{tabular}{|l|} 
myristoleoylcarnitine \\
\end{tabular} & 0.73 & 0.64 \\
\hline & 3-hydroxybutyrate & 1.22 & 2.91 \\
\hline & 3-hydroxysuberate & 0.94 & 1.97 \\
\hline & 3-hydroxyhexanoate & 1.01 & 195 \\
\hline \multirow{5}{*}{ Free Fatty Acids } & linolenate & 0.63 & 0.53 \\
\hline & tetradecadienoate & 0.73 & 0.55 \\
\hline & \begin{tabular}{|l|} 
stearidonate \\
\end{tabular} & 0.72 & 0.62 \\
\hline & hexadecatrienoate & 0.68 & 0.65 \\
\hline & linoleate & 0.74 & 0.68 \\
\hline \multicolumn{4}{|l|}{ Amino Acids } \\
\hline \multirow{5}{*}{ (Anaplerosis) } & cysteine & 1.56 & 3.22 \\
\hline & alanine & 1.32 & 1.63 \\
\hline & proline & 1.1 & 1.38 \\
\hline & leucine & 1.05 & 1.29 \\
\hline & serine & 1.12 & 1.28 \\
\hline \multirow{5}{*}{$\begin{array}{l}\text { (Branch-Chain } \\
\text { Amino Acids) }\end{array}$} & tigloyglycine & 1.67 & 18.61 \\
\hline & 3-methylcrotonylglycin & 1.65 & 9.72 \\
\hline & \begin{tabular}{|l|} 
2-methylbutyrylglycine \\
\end{tabular} & 1.44 & 6.49 \\
\hline & $\begin{array}{l}\text { 2,3-dihydroxy-2- } \\
\text { mrthylbutyrate }\end{array}$ & 1.85 & 6.01 \\
\hline & isovalerylglycine & 1.78 & 5.1 \\
\hline
\end{tabular}

(cont.)

\begin{tabular}{|c|c|c|c|}
\hline \multirow{5}{*}{$\begin{array}{l}\text { (N-acetyl Amino } \\
\text { Acids) }\end{array}$} & $\mathrm{N}$-acetlytyrosine & 1.54 & 3.91 \\
\hline & $\mathrm{N}$-acetylleucine & 1.59 & 3.88 \\
\hline & $\mathrm{N}$-acetylisoleucine & 1.52 & 3.32 \\
\hline & $\mathrm{N}$-acetylglutamate & 1.26 & 3.14 \\
\hline & $\mathrm{N}$-acetylglutamine & 1.26 & 3.04 \\
\hline \multirow{2}{*}{ (Acetylated Peptides) } & phenylacetylglycine & 1.43 & 4.77 \\
\hline & phenylacetyltaurine & 2.34 & 7.63 \\
\hline \multirow{5}{*}{$\begin{array}{c}\text { (Carboxyethyl Amino } \\
\text { Acids) }\end{array}$} & 1-carboxyethyltyrosine & 1.68 & 6.65 \\
\hline & 1-carboxyethylisoleucine & 1.99 & 6.02 \\
\hline & 1-carboxyethylyaline & 1.88 & 5.55 \\
\hline & $1-$ & 1.6 & 4.96 \\
\hline & 1-carboxyethylleucine & 1.99 & 4.95 \\
\hline \multirow{5}{*}{ (Acylglycines) } & 3-hydroxyoctanoylglycin & 1.11 & 13.81 \\
\hline & trans-2-hexanoylglycine & 0.93 & 12.79 \\
\hline & 2-butenoylglycine & 1.23 & 11.44 \\
\hline & 3-hydroxybutyroylglycin & 1.5 & 8 \\
\hline & isocaproylglycine & 1.49 & 2.8 \\
\hline \multirow{5}{*}{$\begin{array}{c}\text { Markers of Protein } \\
\text { Homeostasis }\end{array}$} & cis-4-hyroxyproline & 1.18 & 2.16 \\
\hline & N2,N6-diacetyllysine & 1.02 & 1.48 \\
\hline & dimethylarginine & 1.1 & 1.46 \\
\hline & leucine & 0.85 & 14 \\
\hline & N6-acetyllycine & 0.96 & 1.38 \\
\hline \multirow{5}{*}{$\begin{array}{l}\text { Glutathione } \\
\text { Metabolism }\end{array}$} & gamma-glutamylcitrullin & 0.54 & 0.47 \\
\hline & gamma-glutamylglutama & 0.47 & 0.54 \\
\hline & gamma-glutamyltryptopl & 0.44 & 0.58 \\
\hline & gamma-glutamylisoleuci & 0.97 & 1.41 \\
\hline & gamma-glutamylvaline & 0.92 & 1.28 \\
\hline \multirow{5}{*}{$\begin{array}{l}\text { Lipid Peroxides and } \\
\text { Eicosanoids }\end{array}$} & 12-HEPE & 0.81 & 0.59 \\
\hline & 14-HDoHE/17-HDoHE & 0.83 & 0.62 \\
\hline & 12-HETE & 0.84 & 0.64 \\
\hline & 13-HODE +9-HODE & 0.81 & 0.73 \\
\hline & 12,13-DiHOME & 0.67 & 0.74 \\
\hline \multirow{3}{*}{ Betaine and Choline } & dimethylglycine & 0.79 & 0.93 \\
\hline & choline & 1.35 & 1.9 \\
\hline & betaine & 0.98 & 1.22 \\
\hline \multirow{3}{*}{$\begin{array}{c}\text { Bile Acid } \\
\text { Metabolism }\end{array}$} & deoxycholate & 2.01 & 3.59 \\
\hline & 7-ketodeoxycholate & 2.25 & 3.52 \\
\hline & taurocholate & 1.56 & 1.8 \\
\hline
\end{tabular}

Fig. 6. The metabolomic effects of IM156 (10 and $30 \mathrm{mg} / \mathrm{kg}$, p.o., daily for 10 days) relative to vehicle are summarized ( $n=10 \mathrm{CD}-1 \mathrm{mice}$ per group). This figure highlights up to five of the most regulated metabolites (all $P<0.05$ ) assigned to metabolic pathways or subpathways. Green signifies a significant $(P<0.05)$ increase and red a significant decrease in a given metabolite compared with vehicle. One-way ANOVA was performed on log-transformed data, and values are expressed as ratios of treatment:vehicle.

associated with regions of fibrosis; occasionally, neutrophilic infiltrates were accompanied by small foci of necrosis in adjacent alveolar septa (Fig. 9B). Pulmonary fibrosis and alveolar damage induced by bleomycin was reduced after delayed treatment with IM156 (Fig. 9C) or pirfenidone (Fig. 9D).

It is noteworthy that a $30 \mathrm{mg} / \mathrm{kg}$ oral dose of IM156 yields a 24-hour area under the curve of $3.06 \mu \mathrm{g} * \mathrm{hr} / \mathrm{ml}$ in the CD-1 mouse, and a well tolerated $200 \mathrm{mg}$ oral dose of IM156 yields a 24 -hour area under the curve of $4.2 \mu \mathrm{g} * \mathrm{hr} / \mathrm{ml}$ in humans (Rha et al., 2020)—demonstrating the relevance of the animal dose range to human exposures (Supplemental Table 3). The percent plasma protein binding of IM156 is 79 and $82 \%$ in rodent and human plasma, respectively.

\section{Discussion}

IPF is the archetypal age-related FILD characterized by a heterogenous decline in lung function (stable or episodic), worsening dyspnea, poor quality of life, and high mortality rate (Cottin et al., 2019). Current antifibrotic standard of care for these patients includes Ofev (nintedanib) and Esbriet (pirfenidone), both of which have been shown to decrease the progression of lung dysfunction and all-cause mortality at 1 year (Graney and Lee, 2018). However, the effects of antifibrotic treatment on survival in these patients are controversial, and reported benefits appear to be less apparent when adjusted for age (Kaunisto et al., 2019). Less impressive are effects on quality of life, as these agents cause significant drug-related adverse events (i.e., diarrhea, nausea, vomiting, abdominal pain, decreased appetite, liver enzyme elevations, rash, weight loss) requiring dose reductions or discontinuance in nearly $50 \%$ of patients at 1 year (Flaherty et al., 2019). Safe and effective novel therapies are urgently needed for patients with FILD.

The regulation of metabolism has become an emerging area of interest in fibrosis research. The transition of a fibroblast, fibrocyte, or pericyte to a myofibroblast, as occurs after TGF $\beta$ stimulation, is a necessary step in fibrogenesis and is associated with a well described metabolic reprogramming needed to support the enhanced energetic and synthetic requirements of the myofibroblast. This metabolic shift is characterized by enhanced aerobic glycolysis, lipogenesis, OXPHOS, and anaplerosis (e.g., glutaminolysis) and is evident in lung regions of active fibrosis in patients with IPF (Win et al., 2012; Bernard et al., 2015; Xie et al., 2015; Bernard et al., 2018; Rabinowitz and Mutlu, 2019; Bueno et al., 2020; Gibb et al., 2020). The mechanisms underlying these metabolic changes are complex and include 


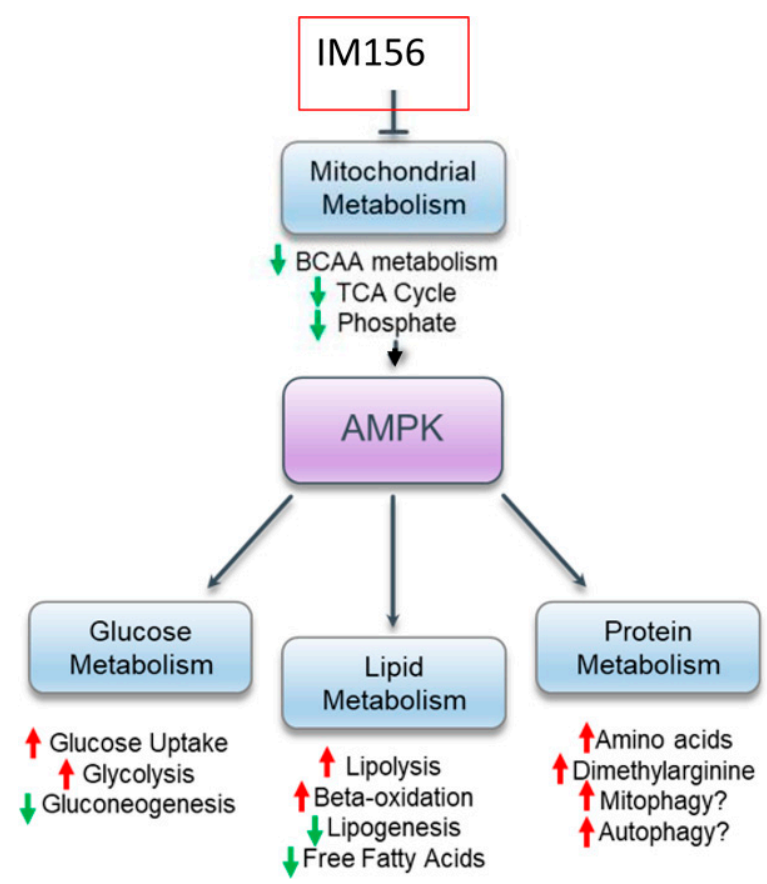

Fig. 7. A pathway model consistent with the metabolomic results suggests IM156 acts at the level of the mitochondria to disrupt OXPHOS and the TCA cycle, adaptively activating AMPK to increase aerobic glycolysis, $\beta$-oxidation, and protein catabolism while decreasing protein synthesis. BCAA (branched chain amino acid).

upregulation of key metabolic transcription factors, transporters, and enzymes, as well as increased mitochondrial mass (Gibb et al., 2020; Hua et al., 2020; Zhao et al., 2020).

A

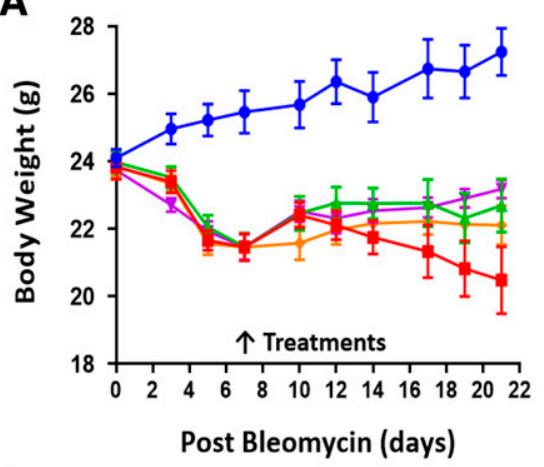

C

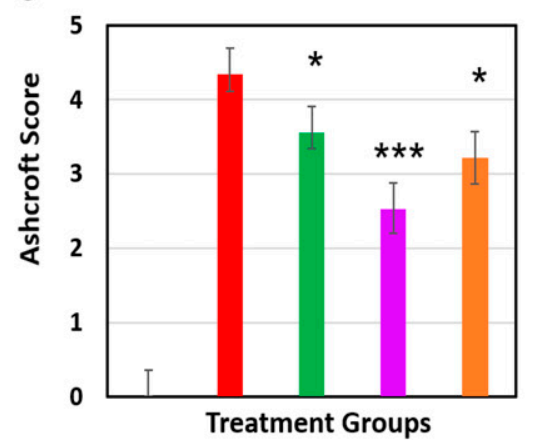

Treatment Groups

$\rightarrow(-)$ BM

- Vehicle + BM

- IM156 $(10 \mathrm{mg} / \mathrm{kg}, q d)+B M$

* IM156 $(30 \mathrm{mg} / \mathrm{kg}, q d)+\mathrm{BM}$

$\rightarrow$ Pirfenidone $(100 \mathrm{mg} / \mathrm{kg}$, bid $)+\mathrm{BM}$

D

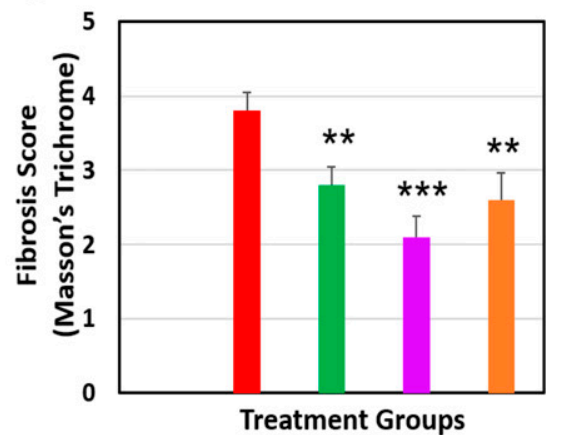

Given the critical role of metabolic reprogramming, it is not surprising that targeting metabolism is now an active area of antifibrotic drug discovery (Zhao et al., 2020). Most metabolic targets have focused on transcriptional modulators [e.g., farnesoid X receptor (FXR), peroxisome proliferator-activated receptor (PPARs)] or glycolytic, lipogenic, and anaplerectic enzymes that have greater or lesser effects in preclinical fibrosis models and have not yet demonstrated clinical efficacy in fibrotic disorders (Cui et al., 2019; Schruf et al., 2019; Smith-Cortinez et al., 2020; Zhao et al., 2020). Less well studied are agents that target modulation of OXPHOS. Metformin, a weak OXPHOS inhibitor, has demonstrated impressive antifibrotic effects in a variety of preclinical studies; however, the clinical translation of these results is questionable given its low potency (Bridges et al., 2014; Shen et al., 2016; Shin et al., 2017; Rangarajan et al., 2018; Izreig et al., 2020) and that evaluations of antifibrotic activity have relied only on retrospective analyses. Based on conservative relative potency comparisons it is estimated that antifibrotic plasma concentration of metformin may need to exceed $12 \mu \mathrm{M}$; however, typical antidiabetic concentrations are in the $2-6 \mu \mathrm{M}$ range (Frid et al., 2010). In the present study we used IM156, a selective PC1 inhibitor, to modulate OXPHOS and characterized its effects on the myofibroblast phenotype, metabolism, and lung fibrosis (Izreig et al., 2020). We found that IM156 had profound metabolic effects and robust antifibrotic activity in the lung at plasma exposures that are clinically relevant and were well tolerated in humans (Rha et al., 2020).

IM156 dose-dependently inhibited TGF $\beta$-dependent increases in mitochondrial oxygen consumption rate and myofibroblast markers (i.e., $\alpha$-SMA, collagen deposition) in human pulmonary

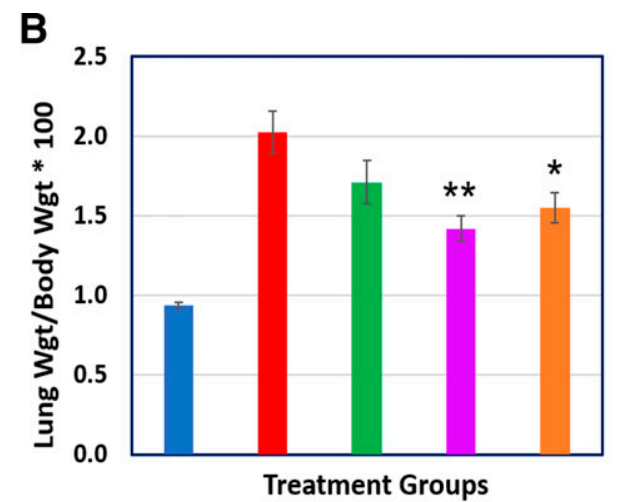

E

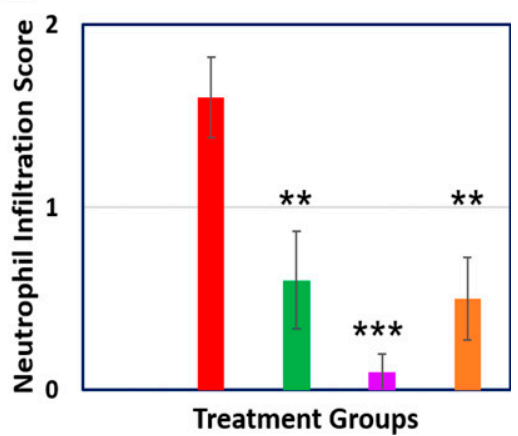

Fig. 8. IM156 was evaluated in the murine bleomycin model of pulmonary fibrosis using a delayed treatment design ( $n=10$ per treatment group). Animals received oropharyngeal administration of bleomycin $(\mathrm{BM} ; 1.5 \mathrm{U} / \mathrm{kg}$ ) on day 0 and were randomized to 14 days of treatment on day 7 . (A) Treatment with IM156 (10 and $30 \mathrm{mg} / \mathrm{kg}$, p.o., every day) and pirfenidone $(100 \mathrm{mg} / \mathrm{kg}$, p.o., twice daily) blunted late weight loss induced by bleomycin as well as associated increases in lung weight (B), fibrosis [(C) and (D)], and inflammation (E). The effects of IM156 were dose-related, and the high-dose group compared favorably with pirfenidone. Values are means \pm S.E.M., and statistical comparisons were performed using a two-way ANOVA with Dunnett's test for analysis of body weight data and a one-way ANOVA with Bonferroni's multiple-comparison test for all other comparisons. ${ }^{*} P<0.05 ; * * P<0.01 ; * * * P<0.001$. 
A Sham Control (No Bleomycin)

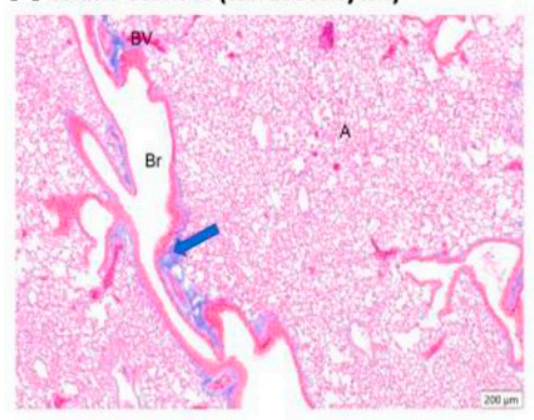

\section{$C_{\text {IM156 }(30 \mathrm{mg} / \mathrm{kg}, q d)}$}

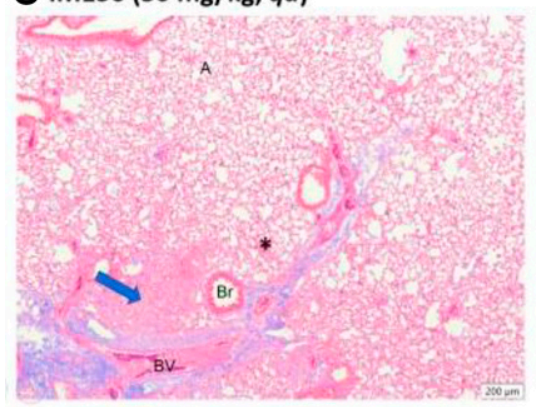

B Vehicle Control

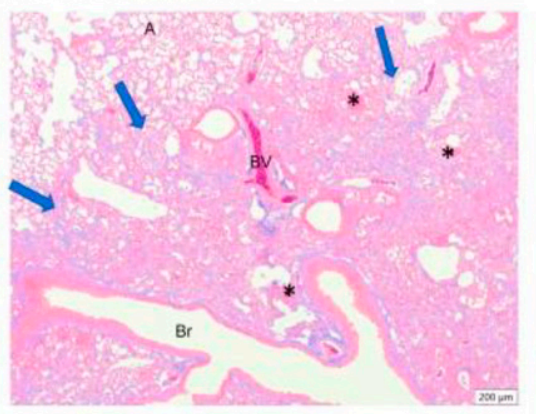

D Pirfenidone $(100 \mathrm{mg} / \mathrm{kg}$, bid $)$

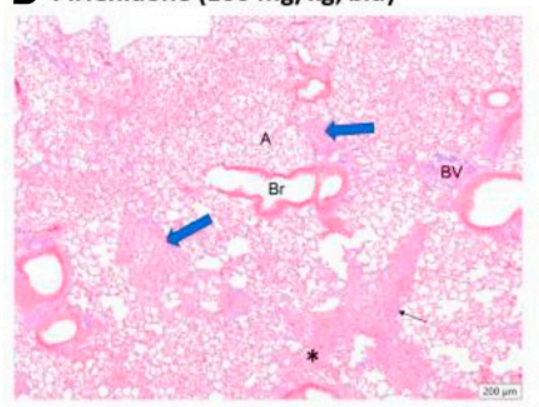

Fig. 9. Representative photomicrographs of paraffin embedded lung sections stained with Masson's trichrome obtained from experimental groups on day 21 after 14 consecutive days of treatment (commencing 7 days after bleomycin treatment). The sham control (A) received the water vehicle only without bleomycin pretreatment. In the bleomycinpretreated vehicle control group (B), the majority of the pulmonary parenchyma was replaced by a contiguous fibrotic mass (borders indicated by arrows) characterized by expansion or obliteration of alveolar septa and markedly increased collagen deposition (blue staining). Asterisks $(*)$ indicate areas of mononuclear cell infiltrates. Pulmonary fibrosis and alveolar damage induced by bleomycin was reduced after delayed treatment with IM156 (C) or pirfenidone (D). A, alveolus; Br, bronchiole; BV, blood vessel. fibroblasts without altering the ECAR beyond what was observed with TGF $\beta$ and had no effect on cell viability. It is likely that the mechanism driving ECAR after TGF $\beta$ alone is not the same as the mechanism driving it in the presence of OXPHOS modulation since both TGF $\beta$ and IM156 increase glycolysis (ECAR) but were not additive.

IM156 was highly distributed to major metabolic peripheral organs (i.e., lung, liver, kidney, heart) after chronic oral administration and had significant dose-related effects on the plasma metabolome, illustrating increases in glycolysis, lipolysis, and $\beta$-oxidation and decreases in TCA cycle activity and protein synthesis. These results demonstrate target engagement consistent with OXPHOS modulation and AMPK activation at clinically relevant exposures. In fact, treatment with IM156 results in robust phosphorylation of AMPK in a number of cellular assays (Ju et al., 2016; Lee et al., 2016) and is approximately 60-fold more potent than metformin (Supplemental Fig. 2). Equivalent doses of IM156 also significantly attenuated systemic changes and lung histopathology (fibrosis and inflammation) in the murine bleomycin-induced model of pulmonary fibrosis, in a dose-related manner, when administered in either prophylactic or delayed treatment paradigms.

The antifibrotic effects of IM156 were not limited to the lung and have been observed in preclinical models of kidney, liver, and peritoneal fibrosis (Ju et al., 2016; Lee et al., 2016; Tsogbadrakh et al., 2018). Furthermore, these results are entirely consistent with recent results in hepatic stellate cells that describe the predominant functional role of OXPHOS in fibrotic metabolic reprogramming (Smith-Cortinez et al., 2020). Also, the lack of an obvious effect of IM156 to inhibit glycolysis is consistent with the failure to block the myofibroblast phenotype by inhibition of glycolysis (Schruf et al., 2019)—suggesting enhanced glycolysis is neither sufficient nor necessary for fibroblast to myofibroblast transition.
The current study provides strong evidence for the role of OXPHOS inhibition and AMPK activation in mediating the antifibrotic effect of IM156 and is consistent with the role of AMPK in the regulation of myofibroblast activation and collagen gene expression (Chen et al., 2014; Kheirollahi et al., 2019). However, future studies will be needed to understand the precise cellular and molecular mechanisms underlying the AMPK-dependent and AMPK-independent antifibrotic effects of OXPHOS modulation with IM156, as well as effects on myofibroblast dedifferentiation and mitochondrial-mediated apoptosis and inflammation (Fortier et al., 2021; Xian et al., 2021). These are currently active areas of research.

In conclusion, our results demonstrate that upregulation of OXPHOS is a necessary metabolic step to support the myofibroblast phenotype and fibrogenesis, and its pharmacological modulation, with a selective PC1 inhibitor (IM156), is a robust antifibrotic mechanism. Early clinical evaluation to test this therapeutic hypothesis in patients with IPF is currently underway.

\section{Acknowledgments}

The authors would like to thank Thomas Richardson for expert discovery chemistry and initial compound characterization, Andrew J. Schwab (Metabolon Inc., Morrisville, NC) for help with analysis of the metabolome and pathway identification, and Rashmi Munshi for assistance with the bleomycin study (Aragen Inc., Morgan Hill, CA).

\section{Authorship Contributions}

Participated in research design: Willette, Cowen, Welsch. Conducted experiments: Mangrolia, Lee, Yoo.

Contributed new reagents or analytic tools: Pondell.

Performed data analysis: Willette, Mangrolia, Yoo, Welsch

Wrote or contributed to the writing of the manuscript: Willette, Rudoltz, Welsch. 


\section{References}

Bernard K, Logsdon NJ, Benavides GA, Sanders Y, Zhang J, Darley-Usmar VM, and Thannickal VJ (2018) Glutaminolysis is required for transforming growth factor- $\beta 1$ induced myofibroblast differentiation and activation. J Biol Chem 293:1218-1228.

Bernard K, Logsdon NJ, Ravi S, Xie N, Persons BP, Rangarajan S, Zmijewski JW, Mitra K, Liu G, Darley-Usmar VM et al. (2015) Metabolic reprogramming is required for myofibroblast contractility and differentiation. J Biol Chem 290:25427-25438.

Bridges HR, Jones AJ, Pollak MN, and Hirst J (2014) Effects of metformin and other biguanides on oxidative phosphorylation in mitochondria. Biochem J 462:475-487.

Brockhoff M, Rion N, Chojnowska K, Wiktorowicz T, Eickhorst C, Erne B, Frank S, Angelini C, Furling D, Rüegg MA et al. (2017) Targeting deregulated AMPK/ mTORC1 pathways improves muscle function in myotonic dystrophy type I. J Clin Invest 127:549-563.

Bueno M, Calyeca J, Rojas M, and Mora AL (2020) Mitochondria dysfunction and metabolic reprogramming as drivers of idiopathic pulmonary fibrosis. Redox Biol 33:101509.

Chen CZ, Peng YX, Wang ZB, Fish PV, Kaar JL, Koepsel RR, Russell AJ, Lareu RR, and Raghunath M (2009) The Scar-in-a-Jar: studying potential antifibrotic compounds from the epigenetic to extracellular level in a single well. $\mathrm{Br} J$ Pharmacol 158:1196-1209.

Chen K-H, Hsu H-H, Lee C-C, Yen T-H, Ko Y-C, Yang C-W, and Hung C-C (2014) The AMPK agonist AICAR inhibits TGF- $\beta 1$ induced activation of kidney myofibroblasts. PLoS One 9:e106554.

Cottin V, Wollin L, Fischer A, Quaresma M, Stowasser S, and Harari S (2019) Fibrosing interstitial lung diseases: knowns and unknowns. Eur Respir Rev 28:180100.

Crissman JW, Goodman DG, Hildebrandt PK, Maronpot RR, Prater DA, Riley JH, Seaman WJ, and Thake DC (2004) Best practices guideline: toxicologic pathology. Toxicol Pathol 32:126-131.

Cui H, Xie N, Jiang D, Banerjee S, Ge J, Sanders YY, and Liu G (2019) Inhibition of glutaminase 1 attenuates experimental pulmonary fibrosis. Am J Respir Cell Mol Biol 61:492-500.

Dykens JA, Jamieson J, Marroquin L, Nadanaciva S, Billis PA, and Will Y (2008) Biguanide-induced mitochondrial dysfunction yields increased lactate production and cytotoxicity of aerobically-poised HepG2 cells and human hepatocytes in vitro. Toxicol Appl Pharmacol 233:203-210.

Evans AM, Bridgewater BR, Liu Q, Mitchell MW, Robinson RJ, Dai H, Stewart SJ, DeHaven CD, and Miller LAD (2014) High resolution mass spectrometry improves data quantity and quality as compared to unit mass resolution mass spectrometry in high-throughput profiling metabolomics. Metabolomics (Los Angel) 4:2.

Flaherty KR, Wells AU, Cottin V, Devaraj A, Walsh SLF, Inoue Y, Richeldi L, Kolb M, Tetzlaff K, Stowasser S et al.; INBUILD Trial Investigators (2019) Nintedanib in progressive fibrosing interstitial lung diseases. $N$ Engl J Med 381:1718-1727.

Fortier SM, Penke LR, King D, Pham TX, Ligresti G, and Peters-Golden M (2021) Myofibroblast dedifferentiation proceeds via distinct transcriptomic and phenotypic transitions. JCI Insight 6:e144799.

Frid A, Sterner GN, Löndahl M, Wiklander C, Cato A, Vinge E, and Andersson A (2010) Novel assay of metformin levels in patients with type 2 diabetes and varying levels of renal function: clinical recommendations. Diabetes Care 33:1291-1293.

Garcia D and Shaw RJ (2017) AMPK: mechanisms of cellular energy sensing and restoration of metabolic balance. Mol Cell 66:789-800

Gibb AA, Lazaropoulos MP, and Elrod JW (2020) Myofibroblasts and fibrosis: mitochondrial and metabolic control of cellular differentiation. Circ Res 127:427-447.

Goldberg HJ (2019) Understanding progressive fibrosing interstitial lung disease through therapeutic trials. N Engl J Med 381:1775-1777.

Graney BA and Lee JS (2018) Impact of novel antifibrotic therapy on patient outcomes in idiopathic pulmonary fibrosis: patient selection and perspectives. Patient Relat Outcome Meas 9:321-328.

Hill BG, Benavides GA, Lancaster Jr JR, Ballinger S, Dell'Italia L, Jianhua Z, and Darley-Usmar VM (2012) Integration of cellular bioenergetics with mitochondrial quality control and autophagy. Biol Chem 393:1485-1512.

Horowitz JC and Thannickal VJ (2006) Epithelial-mesenchymal interactions in pulmonary fibrosis. Semin Respir Crit Care Med 27:600-612.

Hua W, Ten Dijke P, Kostidis S, Giera M, and Hornsveld M (2020) TGF $\beta$-induced metabolic reprogramming during epithelial-to-mesenchymal transition in cancer. Cell Mol Life Sci 77:2103-2123.

Hübner RH, Gitter W, El Mokhtari NE, Mathiak M, Both M, Bolte H, Freitag-Wolf S, and Bewig B (2008) Standardized quantification of pulmonary fibrosis in histological samples. Biotechniques 44:507-511, 514-517.

Izreig S, Gariepy A, Kaymak I, Bridges HR, Donayo AO, Bridon G, DeCamp LM, Kitchen-Goosen SM, Avizonis D, Sheldon RD et al. (2020) Repression of LKB1 by miR-17 92 sensitizes MYC-dependent lymphoma to biguanide treatment. Cell Rep Med 1:100014.

Ju KD, Kim HJ, Tsogbadrakh B, Lee J, Ryu H, Cho EJ, Hwang YH, Kim K, Yang J, Ahn C et al. (2016) HL156A, a novel AMP-activated protein kinase activator, is protective against peritoneal fibrosis in an in vivo and in vitro model of peritoneal fibrosis. Am J Physiol Renal Physiol 310:F342-F350.
Kaunisto J, Salomaa E-R, Hodgson U, Kaarteenaho R, Kankaanranta H, Koli K, Vahlberg T, and Myllärniemi M (2019) Demographics and survival of patients with idiopathic pulmonary fibrosis in the FinnishIPF registry. ERJ Open Res 5:00170-2018.

Kheirollahi V, Wasnick RM, Biasin V, Vazquez-Armendariz AI, Chu X, Moiseenko A, Weiss A, Wilhelm J, Zhang J-S, Kwapiszewska G et al. (2019) Metformin induces lipogenic differentiation in myofibroblasts to reverse lung fibrosis. Nat Commun 10:2987.

Kolb M and Vašáková M (2019) The natural history of progressive fibrosing interstitial lung diseases. Respir Res 20:57.

Kuppe C, Ibrahim MM, Kranz J, Zhang X, Ziegler S, Perales-Patón J, Jansen J, Reimer KC, Smith JR, Dobie R et al. (2021) Decoding myofibroblast origins in human kidney fibrosis. Nature 589:281-286.

Lee HS, Shin HS, Choi J, Bae SJ, Wee HJ, Son T, Seo JH, Park JH, Kim SW, and Kim KW (2016) AMP-activated protein kinase activator, HL156A reduces thioacetamide-induced liver fibrosis in mice and inhibits the activation of cultured hepatic stellate cells and macrophages. Int J Oncol 49:1407-1414.

Li Y and Chen Y (2019) AMPK and Autophagy, in Autophagy: Biology and Diseases: Basic Science (Qin Z-H, ed) pp 85-108, Springer Singapore, Singapore.

Meng XM, Nikolic-Paterson DJ, and Lan HY (2016a) TGF- $\beta$ : the master regulator of fibrosis. Nat Rev Nephrol 12:325-338.

Oku H, Shimizu T, Kawabata T, Nagira M, Hikita I, Ueyama A, Matsushima S, Torii M, and Arimura A (2008) Antifibrotic action of pirfenidone and prednisolone: different effects on pulmonary cytokines and growth factors in bleomycin-induced murine pulmonary fibrosis. Eur J Pharmacol 590:400-408.

Rabinowitz JD and Mutlu GM (2019) A metabolic strategy to reverse fibrosis? Nat Metab 1:12-13.

Rangarajan S, Bone NB, Zmijewska AA, Jiang S, Park DW, Bernard K, Locy ML, Ravi S, Deshane J, Mannon RB et al. (2018) Metformin reverses established lung fibrosis in a bleomycin model. Nat Med 24:1121-1127.

Redente EF, Jacobsen KM, Solomon JJ, Lara AR, Faubel S, Keith RC, Henson PM, Downey GP, and Riches DWH (2011) Age and sex dimorphisms contribute to the severity of bleomycin-induced lung injury and fibrosis. Am J Physiol Lung Cell Mol Physiol 301:L510-L518

Rha SY, Beom S-H, Shin YG, Yim D-S, Moon YW, Kim TW, Kim SY, Kim GM, Kim HS, Cheong J-H et al (2020) Phase I study of IM156, a novel potent biguanide oxidative phosphorylation (OXPHOS) inhibitor, in patients with advanced solid tumors (Abstract). J Clin Oncol 38(suppl 15):3590.

Schruf E, Schroeder V, Kuttruff CA, Weigle S, Krell M, Benz M, Bretschneider T, Holweg A, Schuler M, Frick M et al. (2019) Human lung fibroblast-to-myofibroblast transformation is not driven by an LDH5-dependent metabolic shift towards aerobic glycolysis. Respir Res 20:87.

Shen Y, Miao N, Xu J, Gan X, Xu D, Zhou L, Xue H, Zhang W, and Lu L (2016) Metformin prevents renal fibrosis in mice with unilateral ureteral obstruction and inhibits Ang IIinduced ECM production in renal fibroblasts. Int $J$ Mol Sci 17:146.

Shin HS, Ko J, Kim DA, Ryu ES, Ryu HM, Park SH, Kim YL, Oh ES, and Kang DH (2017) Metformin ameliorates the phenotype transition of peritoneal mesothelial cells and peritoneal fibrosis via a modulation of oxidative stress. Sci Rep 7:5690.

Smith-Cortinez N, van Eunen K, Heegsma J, Serna-Salas SA, Sydor S, Bechmann LP, Moshage H, Bakker BM, and Faber KN (2020) Simultaneous induction of glycolysis and oxidative phosphorylation during activation of hepatic stellate cells reveals novel mitochondrial targets to treat liver fibrosis. Cells 9:2456.

Stewart AG, Thomas B, and Koff J (2018) TGF- $\beta$ : master regulator of inflammation and fibrosis. Respirology 23:1096-1097.

Tsogbadrakh B, Ju KD, Lee J, Han M, Koh J, Yu Y, Lee H, Yu KS, Oh YK, Kim HJ et al. (2018) HL156A, a novel pharmacological agent with potent adenosine-monophosphate-activated protein kinase (AMPK) activator activity ameliorates renal fibrosis in a rat unilateral ureteral obstruction model. PLoS One 13:e201692.

Walters D and Kleeberger, SR (2008) Mouse models of bleomycin-induced pulmonary fibrosis. Curr Protoc Pharmacol 5:46.

Win T, Lambrou T, Hutton BF, Kayani I, Screaton NJ, Porter JC, Maher TM, Endozo R, Shortman RI, Lukey P et al. (2012) 18F-Fluorodeoxyglucose positron emission tomography pulmonary imaging in idiopathic pulmonary fibrosis is reproducible: implications for future clinical trials. Eur J Nucl Med Mol Imaging 39:521-528.

Xian H, Liu Y, Rundberg Nilsson A, Gatchalian R, Crother TR, Tourtellotte WG, Zhang Y, Aleman-Muench GR, Lewis G, Chen W et al. (2021) Metformin inhibition of mitochondrial ATP and DNA synthesis abrogates NLRP3 inflammasome activation and pulmonary inflammation. Immunity 54:1463-1477.e11.

Xie N, Tan Z, Banerjee S, Cui H, Ge J, Liu RM, Bernard K, Thannickal VJ, and Liu G (2015) Glycolytic reprogramming in myofibroblast differentiation and lung fibrosis. Am J Respir Crit Care Med 192:1462-1474.

Yazdani S, Bansal R, and Prakash J (2017) Drug targeting to myofibroblasts: implications for fibrosis and cancer. Adv Drug Deliv Rev 121:101-116.

Zent J and Guo LW (2018) Signaling mechanisms of myofibroblastic activation: outside-in and inside-out. Cell Physiol Biochem 49:848-868.

Zhao X, Kwan JYY, Yip K, Liu PP, and Liu FF (2020) Targeting metabolic dysregulation for fibrosis therapy. Nat Rev Drug Discov 19:57-75.

Address correspondence to: Dr. Robert N. Willette, ImmunoMet Therapeutics, 2450 Holcombe Blvd., Houston, TX 77021. E-mail: bwillette@ immunomet.com 